\title{
A systematic review and meta-analysis to reappraise the role of adjuvant hepatic arterial infusion for colorectal cancer liver metastases
}

\author{
Richard Lawrence Nelson ${ }^{1}$ (D)
}

Accepted: 7 March 2016/Published online: 12 March 2016

(C) Springer-Verlag Berlin Heidelberg 2016

Dear Editor:

Several months ago, a systematic review of by Liu et al. entitled:

A systematic review and meta-analysis to reappraise the role of adjuvant hepatic arterial infusion for colorectal cancer liver metastases was published in the Int. J. Colorectal Disease vol 30. pp 1091-1102.

Liu found in his meta-analysis that hepatic artery chemotherapy given after resection of liver metastases from colorectal cancer improved overall survival. This review contains a mixture of randomized trials and nonrandomized studies and his findings demonstrate the danger of such a combination. In the Cochrane review of this same topic [1] (not cited), which contained only randomized trials, there was no demonstrable benefit to this intervention. In fact there was an insignificant survival benefit for the control group. Five of the nine studies in the Liu article were non-randomized and in the Cochrane review there were seven randomized trials (eight in the update currently in preparation).

Sincerely yours,

Richard Nelson

\section{References}

1. Nelson RL, Freels S (2006) Hepatic artery adjuvant chemotherapy for patients having resection or ablation of colorectal cancer metastatic to the liver. Cochrane Database Syst Rev. Issue 4. Art. No.: CD003770. doi:10.1002/14651858.CD003770.pub3

Richard Lawrence Nelson

altohorn@uic.edu 